\title{
Organ Culture Model of Liver for the Study of Cancer Treatment for Hepatocellular Carcinoma
}

\author{
Torricelli Piera $^{1}$, Antonelli Francesco ${ }^{2}$, Ferorelli Pasquale ${ }^{2}$, De Martino Angelo ${ }^{2}$, Shevchenko Anna ${ }^{3}$, \\ Siciliano Alberto ${ }^{2}$, Beninati Simone ${ }^{2, "}$ \\ ${ }^{1}$ Department SPES, University of Molise, Campobasso, Italy \\ ${ }^{2}$ Department of Biology, University of Tor Vergata, Rome, Italy \\ ${ }^{3}$ People's Friendship University of Russia, Moscow, Russia
}

Email address:

beninati@bio.uniroma2.it (Beninati S.)

${ }^{*}$ Corresponding author

\section{To cite this article:}

Torricelli Piera, Antonelli Francesco, Ferorelli Pasquale, De Martino Angelo, Shevchenko Anna, Siciliano Alberto, Beninati Simone. Organ Culture Model of Liver for the Study of Cancer Treatment for Hepatocellular Carcinoma. Cancer Research Journal.

Vol. 4 No. 2, 2016, pp. 37-42. doi: 10.11648/j.crj.20160402.13

Received: February 22, 2016; Accepted: March 1, 2016; Published: March 16, 2016

\begin{abstract}
The liver, the largest organ of the human body, is a multifunctional organ with various metabolic activities that plays a fundamental role in maintaining the body and in sustaining life. Although the liver has great regenerative capacity and recovery, the damage caused by chronic diseases such as cancer or viral infections can lead to permanent loss of liver function. Studies on the mechanism of liver disease, have focused on the selection of cell and tissue culture techniques, including strategies based on in vitro models. The organ culture is a promising tool for the study of liver diseases, because it can mimic the complex of the microenvironment in vivo using a three-dimensional model of human liver tissue. These models allow a better study of the specific functions of the liver. In this context, we have analyzed the development of a hepatocarcinoma, obtained by inoculating a murine hepatocarcinoma cell line, Hepa 1/A1s, in the liver of 10 mice of the strain C57BL / 6. After 20 days from the inoculation, the portion of liver invaded by the tumor was removed from the animals and cultured. A group of 5 liver explants were used as a control and other 5 explants were cultured for 4 weeks in a complete medium containing $10 \%$ Citozym, a food supplement with reported antioxidant properties. The cancer-invaded hepatic lobes, treated with Citozym, showed a clear reduction of the weight and the volume of the hepatic tumors, when compared with the control explants.
\end{abstract}

Keywords: Food Supplements, Antioxidants, Hepatocarcinoma, Organ Cultures, Oxidative Stress

\section{Introduction}

Cirrhosis and hepatocellular carcinoma (HCC) have been put to the fifth most common cause of death. Liver cirrhosis is the bridge between the chronic inflammation of the liver to $\mathrm{HCC}$, therefore any kind of chronic inflammatory disease that affects this organ, such as viral hepatitis or alcoholic hepatitis can lead to this form of cancer [1]. For HCC new diagnostic and therapeutic methods were introduced and validated, and many more on the horizon are planned. The future should bring us increasingly effective therapies on cancer and less harmful to the cirrhotic liver, possibly associated with the use of new experimental approaches, ensuring greater availability of antineoplastic drugs possibly characterized by low cytotoxicity. The animal models used for the trials lead to interesting results, but refuted by many, both for the use of animals and for the results considered not comparable with those obtainable on the human [2]. Furthermore, suggestion to use primary liver cells in culture is not justified, because the isolated cell does not behave like that present in the parenchyma of the organ [3]. Social pressures are also focusing on reducing the use of animals and then ultimately ask the replacement of animal testing with alternative models. However, a variety of in vitro models, explored over the last decade, have not been sufficiently positive to be able to replace the animal models. New initiatives seek to address this problem by funding the development of physiologically relevant models of human organs [4]. In this context some 
success is getting the organ culture, as far as the difficulties of maintaining and treatment of the organ still present serious difficulties. The field of medical therapy is particularly the one that most needs to develop. Within this scenario, the development should be, rather than by conventional therapy, with use of new experimental approaches, such as angiogenesis inhibitors and cell differentiation on organs in culture. The same methods used for the organ culture can be used to maintain in culture tumor explants of different origin [5]. These explants can be biopsies of surgical ablation of tumor masses. Following the histological characterization of the tumor, the biopsy sample is grown in suitable condition to maintain proper oxygenation and nutrition. This model allows experiencing cytotoxic and / or cytostatic agents to evaluate, the potential antineoplastic activity in a natural environment.

The March 29, 2014 came into force in Italy the new Legislative Decree no. 26/2014 on animal experiments. This prohibits from 1 January 2017, the use of animals for experimentation and in particular to use animals in experiments with the level of severe pain. It will be promoted and adopted alternative methods / replacement vivisection because there will be a fund for their development, accounting for 50 percent of the revolving fund of the State referred to in Article 5 of Law 16 April 1987, n. 183. All of this leads to the search of alternatives to animal testing. Furthermore it is evident the positive impact on the public, of supplements or drugs, testing of which has been carried out without the use of animals.

Our project aims has this end, that use the organ culture with experimental models are very close to humans, and therefore definitely more reliable from a scientific perspective.

Among many factors, that cause cancer, oxidative stress is one of the most important and well-studied event that gives rise to the conditions leading to tumor onset and progression [6]. The main cause of the mechanism of oxidative stress, are free radicals, substances with high chemical reactivity. Living organisms tend to keep constant the concentration of these oxidizing agents, in order to ensure normal biological processes.

Oxidative stress damages the structures of the cell membrane, in particular the pump ATPase sodium / potassium. It implements therefore, a depolarization and a greater alteration of the active transport mechanism of these two electrolytes, which have very different and essential functions in cells. In this way, this pump, checks the modification of the acid-base reactions and oxidation-reduction between the molecules present in the cytoplasm [7]. It is well known, that there is an antagonism between sodium and potassium in that the more rapid the growth, the greater is the proportion of potassium. [8], which increases during the cell degeneration. In addition, to actively divide, the neoplastic cell, burns high amounts of glucose. These processes of accumulation of glucose, affect cellular respiration, with reduction of oxidative phosphorylation and substantial increase in anaerobic glycolysis. It is then also enhanced the production of lactic acid, formed by reduction from pyruvate. Pyruvate is an inhibitor of the entry into $\mathrm{S}$ phase of mitosis and its steady decline in the cytoplasm (for conversion into lactic acid), it removes the block the mitosis, pushing the cell to an uncontrolled proliferation.

Our objective was to test the antineoplastic potential of an antioxidant called Citozym (CIZ) [9] on a HCC experimentally induced in a mouse liver, explanted and cultured for several days.

\section{Experimental Protocol}

\subsection{Animal Treatments and Liver Cultures}

Male mice C57BL / 6 were obtained from Charles River (Sulzfeld, Germany). The mice were housed with controlled temperature and humidity and with a cycle of $12 \mathrm{~h}$ light / dark. Food and water ad libitum (Harlan Laboratories BV, Horst, the Netherlands). The animals were acclimated for at least seven days before the start of the experiment. All experiments were approved by the ethics committee concerned (n. CD23471N18 project). The experimental procedures were carried out following the guidelines proposed by the National Italian Research Council (CNR), and the National Institute of Health (ISS). Two groups of 5 male mice each were treated for induction of a hepatocarcinoma by intraperitoneal injection, directly into the liver, of $150 \mu \mathrm{L}$ of a cells suspension $\left(10^{5}\right.$ cells) of the murine HCC cell line, Hepa 1/A1s. Each group was then reared in separate cages for 20 days and checked daily. A partial hepatectomy was performed at day $21^{\text {st }}$ from cancer cells injection. Explants were placed onto a $35-\mathrm{mm}$ Greiner dishes - petri dish, 35 x $10 \mathrm{~mm}$, triple vented (Sigma-Aldrich, USA), floating on $7 \mathrm{~mL}$ of a defined serum-free medium and incubated for 28 days at $37^{\circ} \mathrm{C}$ in a humidified incubator $\left(5 \% \quad \mathrm{CO}_{2}\right)$. Defined medium composition, was DMEM/F12 supplemented with $15 \mathrm{mM}$ HEPES, sodium bicarbonate $45 \mathrm{mM}$ ( $\mathrm{pH}$ 7.45) and transferrin $6 \mathrm{mM}$, selenium $7 \mathrm{mM}$, insulin $8 \mathrm{mM}$, triiodothyronine $2 \mathrm{mM}$, prostaglandin $\mathrm{E}_{1} 6 \mathrm{mM}$ and 100 $\mathrm{IU} / \mathrm{mL}$ of Penicillin and $100 \mathrm{ug} / \mathrm{mL}$ of Streptomycin. The culture medium, for the treated explants, was supplemented with $10 \% \mathrm{CIZ}$, while the control group was treated with $10 \%$ sucrose. Complete culture medium was changed daily and no fungicide was present throughout the experiment. CIZ was obtained from CITOZEATEC, S.r.l. (Peschiera Borromeo, Milano, Italy). The main components of CIZ are as follows (units/100 g): $500 \mathrm{mg}$ of vitamin C; $56 \mathrm{mg}$ of vitamin B5; 56 $\mu \mathrm{g}$ of vitamin $\mathrm{D} ; 3,3 \mathrm{mg}$ of vitamin B9; $222 \mathrm{mg}$ of pyruvic acid; $120 \mathrm{mg}$ of citric acid; $250 \mathrm{mg}$ of tartaric acid and $77.8 \mathrm{~g}$ of carbohydrates.

\subsection{Surgery}

For resection, a previously reported technique and perioperative management was performed [10]. Briefly, an upper midline incision was executed from the xyphoid. The liver was gently mobilized by dissecting the falciform ligament and the lobes were exposed and resected close to the inferior vena cava. 


\subsection{Histopathological and Immunohistochemical Analyses}

Regarding the HCC model, appearance of tumor, intrahepatic invasion, peritoneal metastasis and tumor weight were evaluated. Tumor weight/liver weight was also calculated. To verify HCC, after careful macroscopic inspection at sacrifice, the livers were removed and fixed overnight in $10 \%$ buffered formalin. Paraffin-embedded sections of livers were prepared by using routine procedures for histopathological and immune histochemical examinations. Maximum sagittal sections of each liver lobe, from one mouse of the two groups, were used for histopathological examination. Four $\mu$ m-thick sections of formalin-fixed and paraffin-embedded livers were prepared and stained with hematoxylin and eosin for histopathology.

The multiplicity of hepatocellular lesions termed foci of cellular alteration, were assessed on a per unit area $\left(/ \mathrm{cm}^{2}\right)$ basis and determined according to the criteria described by Frith et al. [11].

\subsection{Bioassays}

Evaluation of the levels of CIZ components in cultured liver explant was carried out analyzing the amount of ascorbic acid and cholecalciferol, as representative markers, in aliquots of the parenchyma of liver explant by HPLC methods [12-14]. Proteins in explants were assayed by a reported method [15].

The eventual loss of lactate dehydrogenase (LDH) from the explants to the medium, was considered as a marker of cell damage. Aliquots of the culture medium (1 $\mathrm{mL})$ were collected daily and tested for LDH. The test was performed using the Cyto Tox-ONE ${ }^{\mathrm{TM}}$ homogeneous Membrane Integrity Assay Kit (Promega, Madison, USA.) The CytoTox-ONE $^{\mathrm{TM}}$ assay is a fluorimetric method for estimating the number of nonviable cells present in multiwall plates. This assay rapidly measures the release of LDH from cells with a damaged membrane. LDH released into the culture medium is measured with a 10-minute coupled enzymatic assay, which results in the conversion of resazurin into a fluorescent resofurin product. The amount of fluorescence produced is proportional to the amount of $\mathrm{LDH}$ release. The fluorescence of the samples was read at $\lambda 590 \mathrm{ex}$ and $\lambda 560 \mathrm{em}$.

\section{Results}

We have shown that cultured liver explants, invaded by HCC, obtained from C57BL / 6 mice inoculated intraperitoneally with cells of the line Hepa 1/A1s, it is sufficiently viable for long times, in order to assess the antineoplastic action of a mixture of antioxidants, present in a nutritional supplement (CIZ). During 4 weeks of culture, the components of CIZ are absorbed by the explants, in varying quantities. As seen in Figure 1, two components used as reference point (Vitamin $\mathrm{C}$ and D) are absorbed, increasingly with time, by the explants. Whereas that vitamin $\mathrm{C}$ has a concentration in the CIZ of $5 \mathrm{mg} / \mathrm{mL}$ and that the amount of CIZ added to the culture medium is $10 \%$, it is possible to consider that, after 4 weeks of culture, it reaches a concentration of ascorbic acid in explants, of about $4 \mu \mathrm{g} / \mathrm{mg}$ of protein. This value represents $25 \%$ of vitamin $\mathrm{C}$ present in the culture medium. The absorption of vitamin D, after 4 weeks, in agreement with the estimate previously calculated, accounted for $37 \%$ in total absorption, reaching in the explant a concentration of about $73 \mathrm{pg} / \mathrm{mg}$ protein (Figure 1).

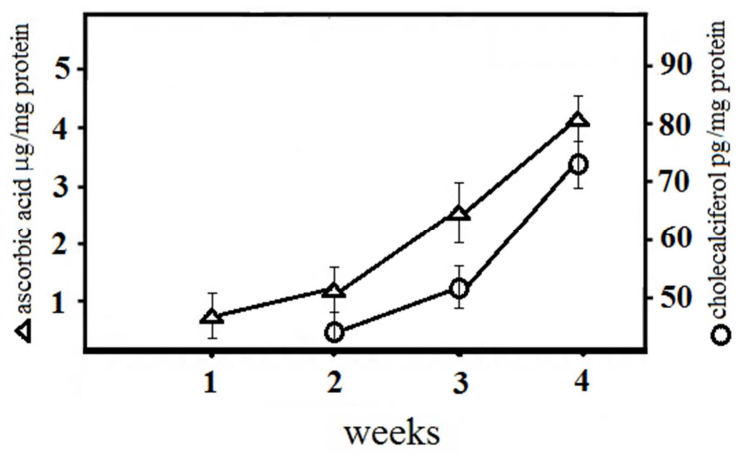

Figure 1. Levels of ascorbic acid and cholecalciferol in cultured liver explants in the presence of CIZ. The value is the average of five determinations $\pm S$. D.

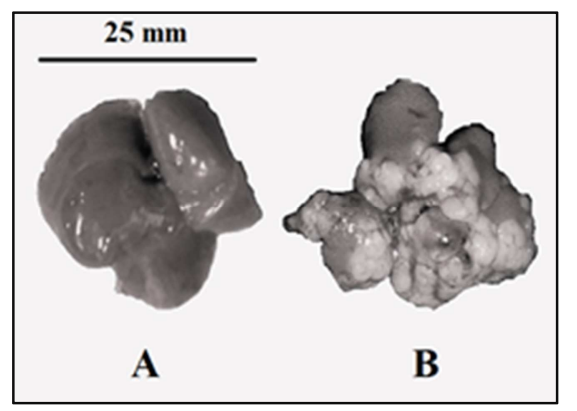

Figure 2. A. liver explant from a mouse not inoculated with Hepa 1/A1s; B. liver explant at day 21st from Hepa 1/A1s cells injection of a CIZ-untreated C57BL/6 mouse.

On day $21^{\text {st }}$ after inoculation of Hepa $1 / \mathrm{A} 1 \mathrm{~s}$ cells, the whole liver explant was occupied by a large HCC mass with scarce normal tissues (Figure 2). The largest tumor nodule was more than $20.0 \pm 2.0 \mathrm{~mm}$ in size. Tumor volume was measured with digital caliper. Microscopic examination of the tumor tissues, demonstrated that normal hepatic structures had been destroyed by invasion of cancer cells (data not shown).

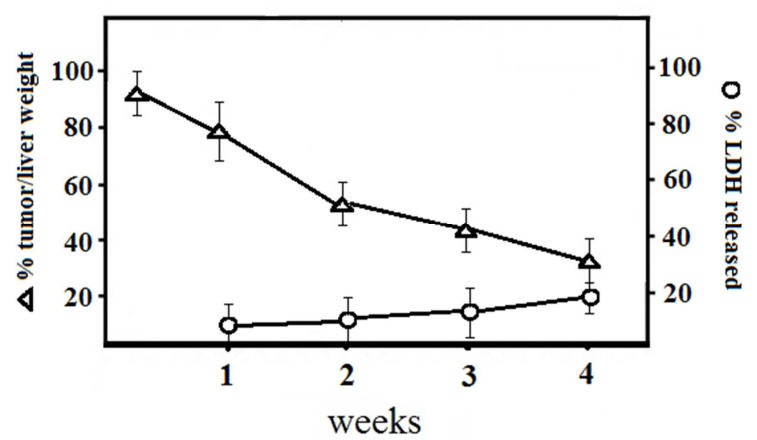

Figure 3. Decrease of tumor/liver weight percentages during the treatment of liver explants with $10 \%$ CIZ as reported in Material and Methods. Levels of $L D H$ released from cultured explants as a marker of cell damage. The value is the average of five determinations $\pm S$. D. 
The reduction of the tumor volume, resulting from the presence of CIZ in the culture medium of the explants, was quantified plotting the weight of the tumor mass in relation to the weight of the liver explant. It is evident the reduction of tumor mass following the treatment with CIZ (Figure 3). Cellular damage, resulting from culture conditions of the liver explants, has remained within acceptable values, as demonstrated by the levels of LDH $(<20 \%)$, released into the culture medium (Figure 3). Figure 4 shows the sharp reduction in the growth of a HCC experimentally induced as indicated in Materials and Methods, in liver explants cultivated in a medium containing $10 \% \mathrm{CIZ}$.

This result was possible thanks to the composition of the culture medium of the explants and continuous oxygenation while in culture.

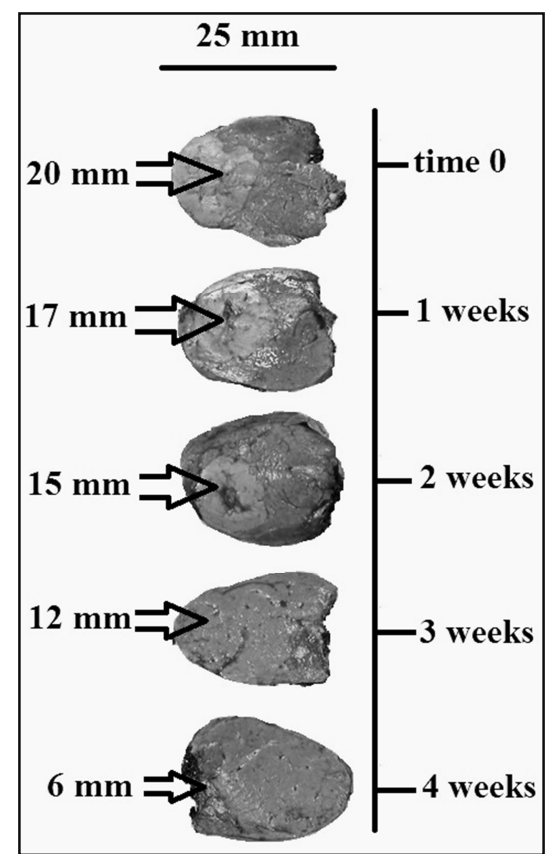

Figure 4. Representative liver explants from C57BL/6 mice, inoculated with cells of the line Hepa 1/A1s during four weeks of incubation in the presence of $10 \% \mathrm{CIZ}$.

\section{Discussion}

HCC has been characterized as a chronic inflammation-driven cancer and the studies of animal models of chemically-induced HCC have revealed the crucial roles of inflammatory signaling in disease onset and severity [16]. A novel animal model of liver cancer (HCC) by hepatoma cell implantation, followed by the culture of HCC invaded explants, was used in this study. The established mouse HCC model is feasible because Hepa $1 / \mathrm{A} 1 \mathrm{~s}$ cells is a mouse hepatoma cell line and it has been demonstrated that Hepa 1/A1s cells can grow in C57BL/6 mouse with the potential of metastasis [17]. We used this model to study the previously reported CIZ activity in anti-inflammation, anti-oxidation and anticancer properties [18].

CIZ, which contains multiple active factors, is an important bioactive dietary supplement. Recently, CIZ has proved useful in reducing the symptoms of various diseases, such as experimental liver steatosis, benign prostatic hyperplasia and antiproliferative activity on estrogen receptor positive and negative human breast adenocarcinoma cell lines [19-21]. The role of the induction of oxidative stress as the mechanism of action of many antitumor drugs is acquiring an increasing interest. In such cases, the antitumor therapy success may be conditioned by the antioxidants present in our own body, which can be synthesized de novo (endogenous) or incorporated through the diet and nutritional supplements (exogenous). Although cells possess a large repertoire of enzymes and antioxidants, sometimes these agents are insufficient to normalize the redox state produced by an intense oxidative stress [22].

In these cases, exogenous antioxidant supplements may be required to restore the cell redox homeostasis [23]. It has been suggested that antioxidant supplementation may protect against oxidative stress associated with the development of certain diseases or that it may reverse the oxidative stress produced during their course. In the area of our interest, that is, cancer, antioxidants are acquiring great importance. It is believed that antioxidants can prevent the development of cancer due to their effects on cell cycle regulation, inflammation, the inhibition of tumor cell proliferation and invasiveness, the induction of apoptosis, and the stimulation of the detoxifying enzyme activity [24].

We have recently reported that CIZ, protected C57BL6/N mice from melanoma carcinogenesis, when chronically given before the initiation and promotion phases of melanoma cancer [9]. The various components of the CIZ, may act synergistically, amplifying the antioxidant and antineoplastic activity of molecules such as pyruvic acid, vitamins $\mathrm{C}$ (ascorbic acid), D3 (cholecalciferol), B5 (pantothenic acid) and B9 (folic acid) complex. Pyruvic acid is an intermediate in the metabolism of glucose and is also a potent scavenger of free radicals. Several studies suggest that pyruvate has potent anti-oxidant and anti-inflammatory actions [25]. In addition, the active form of vitamin D3, $1 \alpha$, 25-dihydroxyvitamin D3 $(1 \alpha, 25(\mathrm{OH}) 2 \mathrm{D} 3$ or calcitriol), is known to inhibit the proliferation and invasiveness of many types of cancer cells, including breast, colon, pancreatic, prostate, and liver cancer cells. [26]. A recent tumor xenograft study, demonstrated that vitamin $\mathrm{C}$ has in vivo an anticancer activity by targeting HIF-1 $\alpha$ [27].

In an interesting series of experiments, Lewisohn and his coworkers [28] reported that the injection of calcium pantothenate along with yeast in mice of the Rill strain led to a considerable reduction in the number of mammary carcinoma transplants. A meta-analysis by Sanjoaquin et al. suggested a protective function of folic acid consumption on colorectal cancer [29]. Our findings show that CIZ significantly inhibits growth of an experimental HCC, investigated by a novel model of organ culture of explants of liver.

Therefore, further in vivo studies, elucidating the mechanisms underlying the activity of this nutraceutical, may be useful in the treatment of tumors and reduction of the side effects of chemotherapy. 


\section{Conclusions}

In our work, we are proposing a new experimental oncology approach, which considers acting with potentially antineoplastic molecules, in an environment very similar to the one which allows the tumor to develop. The application of this method, has been tested using a dietary supplement, with antioxidant components, which has shown strong anticancer properties.

\section{Acknowledgements}

We are grateful to A. I. A. S. Italy, for financial support for studying the possibility of alternative therapies for cancer.

\section{References}

[1] Cardin R, Piciocchi M, Bortolami M, Kotsafti A, Barzon L, Lavezzo, SinigagliaA, Rodriguez-Castro KI, Rugge M, Farinati F. Oxidative damage in the progression of chronic liver disease to hepatocellular carcinoma: an intricate pathway. WorldJ Gastroenterol. 2014; 20(12): 3078-3086.

[2] Langley, G. La validité de l'expérimentation animale en recherche médicale. Revue Semestrielle de Droit Animalier RSDA. 2009; 1: 161-168.

[3] Paixão RL, Schramm FR. Ethics nd animal experimentation: what is debated? Cad Saude Publica. 1999; 15 Suppl 1: 99-110.

[4] Edelman LB, Eddy JA, Price ND. In silico models of cancer. Wiley Interdiscip Rev Syst Biol Med. 2010; 2(4): 438-459.

[5] Carranza-Torres IE, Guzmán-Delgado NE, Cornado-Martínez C, Bañuelos-García JI, Viveros-Valdez E, Morán-Martínez J, Carranza-Rosales P. Organotypic culture of breast tumor explants as a multicellular system for the screening of natural compounds with antineoplastic potential. Biomed Res Int. 2015: 618021.

[6] Sosa V., Moliné T., Somoza R., Paciucci R., Kondoh H., L Leonart M. E. Oxidative stress and cancer: an overview. Ageing Research Reviews. 2013; 12(1): 376-390.

[7] Howarth C, Gleeson P, Attwell D. Updated energy budgets for neural computation in the neocortex and cerebellum. J. Cereb. Blood Flow Metab.2012; 32 (7): 1222-1232.

[8] Wang HY, O'Doherty GA. Modulators of Na/K-ATPase: a patent review. Expert Opin Ther Pat. 2012; 22(6): 587-605.

[9] Antonelli F and Beninati S. Enhanced survival of B16-F10 melanoma tumour-bearing C57BL6/N mice treated with a mixture of antioxidants in: Recent Res Devel in Life Sci. Research Signpost, Trivandrum India.2011; 5: 51-60.

[10] Greene AK, Puder M. Partial hepatectomy in the mouse: technique and perioperative management. J Invest Surg. 2003; 16(2): 99-102.

[11] Frith CH, Ward JM, Turusov VS. Tumours of the liver. IARCSci Publ. 1994; (111): 223-269.

[12] Moreno P, Salvadó V. Determination of eight water- and fat-soluble vitamins in multi-vitamin pharmaceutical formulations by high-performance liquid chromatography. J Chromatogr A. 2000; 870(1-2): 207-215.
[13] Romero Rodriguez MA, Vazquez Oderiz ML, Lopez Hernandez J, Simal Lozano J. Determination of vitamin C and organic acids in various fruits by HPLC. J Chromatogr Sci. 1992; 30(11): 433-437.

[14] Spínola V, Llorent-Martínez EJ, Castilho PC. Determination of vitamin $\mathrm{C}$ in foods: current state of method validation. $\mathrm{J}$ Chromatogr A. 2014; 1369: 2-17.

[15] Bradford, M. M. Rapid and sensitive method for the quantitation of microgram quantities of protein utilizing the principle of protein-dye binding, Anal. Biochem. 1976; 72: 248-254.

[16] Maeda S, Kamata H, Luo JL, Leffert H, Karin M, et al. IKK beta couples hepatocyte death to cytokine-driven compensatory proliferation that promotes chemical hepatocarcinogenesis. Cell. 2005; 121: 977-990.

[17] Kroger A, Ortmann D, Krohne TU, et al. Growth suppression of the hepatocellular carcinoma cell line Hepal-6 by an activatable interferon regulatory factor-1 in mice. Cancer Res. 2001; 61: 2609-2617.

[18] Torricelli P, Ferorelli P, De Martino A, Antonelli F, A. Shevchenko A, Beninati S. Regression of Carotid Plaques in Individuals at Low-to-intermediate Cardiovascular Risk Treated with Citozym and Propulzym. European Journal of Preventive Medicine. 2014; 2 (3): 33-37.

[19] Torricelli P., Ferorelli P., De Martino A., Antonelli F., Beninati S. The influence of preventive multiple micronutrients supplementation on liver steatosis in high-cholesterol fed C57BL6/N mice. American J Life Sciences, 2013; 1(2): 55-60.

[20] Torricelli P. Ferorelli P., De Martino A., Antonelli F., Beninati S. Preventive effects of a mixture of micronutrients with antioxidative properties on experimentally induced prostate hyperplasia. American J. Life Sciences, 2013; 1(1): 22-26.

[21] Torricelli P., Antonelli F., Ferorelli P., De Martino A., Shevchenko A., Beninati S. Antiproliferative activity of a dietary supplement on estrogen receptor positive and negative human breast adenocarcinoma cell lines. Cancer Research J 2014; 2(2): 29-32.

[22] Ozben T. Antioxidant supplementation on cancer risk and during cancer therapy: an update. Current Topics in Medicinal Chemistry. 2015; 15(2): 170-178.

[23] Radimer K., Bindewald B., Hughes J., Ervin B., Swanson C., Picciano M. F. Dietary supplement use by US adults: data from the National Health and Nutrition Examination Survey, 1999-2000. The American Journal of Epidemiology. 2004; 160(4): 339-349.

[24] Bennett L. L., Rojas S., Seefeldt T. Role of antioxidants in the prevention of cancer. Journal of Experimental and Clinical Medicine. 2012; 4(4): 215-222.

[25] Undurti N. Das. Pyruvate is an endogenous anti-inflammatory and anti-oxidant molecule Med Sci Monit, 2006; 12(5): RA79-84.

[26] Matsumoto Y, Kittaka A, Chen TC. 19- Norvitamin D analogs for breast cancer therapy. Can J Physiol Pharmacol. 2015; 93(5): 333-348. 
[27] Gao P, Zhang H, Dinavahi R, Li F, Xiang Y, Raman V, Bhujwalla ZM, Felsher DW, Cheng L, Pevsner J, Lee LA, Semenza GL, Dang CV. HIF-dependent antitumorigenic effect of antioxidants in vivo. Cancer Cell. 2007; 12(3): 230-238.

[28] Lewisohn R. Leuchtenberger C., Leuchtenberger R Laszlo D., and Bloch, K. Prevention of Tumor Growth (Carcinoma 2163) by Intravenous Injections of Yeast and Vitamins. Science 1941; 94: $70-71$

[29] Sanjoaquin M. A., Allen N., Couto E., Roddam A. W. and Key T. J. Folate intake and colorectal cancer risk: a me ta-analytical approach. International journal of cancer. Journal international du cancer. 2005; 113, 825-828. 